\title{
Article \\ Effects of Synaptic Pruning on Phase Synchronization in Chimera States of Neural Network
}

\author{
Zhengyuan Zhang ${ }^{1,2}$ and Liming Dai ${ }^{1,2, *}$ \\ 1 Sino-Canada Nonlinear Science and Complex Systems Center, Wuhan Polytechnic University, \\ Wuhan 430023, China; zze263@uregina.ca \\ 2 Industrial Systems Engineering, University of Regina, Regina, SK S4S 0A2, Canada \\ * Correspondence: liming.dai@uregina.ca; Tel.: +1-306-585-4498
}

check for

updates

Citation: Zhang, Z.; Dai, L. Effects of Synaptic Pruning on Phase

Synchronization in Chimera States of Neural Network. Appl. Sci. 2022, 12, 1942. https://doi.org/10.3390/ app12041942

Academic Editors: Roman Starosta and Jan Awrejcewicz

Received: 30 December 2021 Accepted: 8 February 2022 Published: 12 February 2022

Publisher's Note: MDPI stays neutral with regard to jurisdictional claims in published maps and institutional affiliations.

Copyright: (C) 2022 by the authors Licensee MDPI, Basel, Switzerland. This article is an open access article distributed under the terms and conditions of the Creative Commons Attribution (CC BY) license (https:// creativecommons.org/licenses/by/ $4.0 /)$.

\begin{abstract}
This research explores the effect of synaptic pruning on a ring-shaped neural network of non-locally coupled FitzHugh-Nagumo (FHN) oscillators. The neurons in the pruned region synchronize with each other, and they repel the coherent domain of the chimera states. Furthermore, the width of the pruned region decides the precision and efficiency of the control effect on the position of coherent domains. This phenomenon gives a systematic comprehension of the relation between pruning and synchronization in neural networks from a new aspect that has never been addressed. An explanation of this mechanism is also given.
\end{abstract}

Keywords: chimera states; synaptic pruning; coupled oscillators

\section{Introduction}

The coexistence of synchronization and desynchronization in complex systems has been attracting attention for a long time in many fields, including neuroscience, physics, cybernetics, and mechanics. Researchers have already noticed that changes in the topology and coupling of the complex system can affect the spatial and temporal distribution of synchronized regions. Though there are some techniques that can be used to control the synchronized regions in complex systems, these techniques are still facing many limitations both in theory and applications.

Neurons are coupled with each other as a network through synaptic connections. In their interaction, most neurons in the network are periodically activated and inhibited, which can be shown from the oscillation of extracellular potential. The frequency of neuron oscillations can vary from less than one hertz to more than one hundred hertz. The phase of a neuron is used to describe the neuron's state, and phase synchronization refers to a large number of neurons discharging at the same time. As one of the most fundamental neural network mechanisms, phase synchronization benefits neural communication and neural plasticity. Therefore, it is involved in many cognitive functions, including the formation of both working memory and long-term memory [1-3].

One of the fundamental phenomena in brain development is synaptic pruning, which refers to the reduction in the amount and density of synapses that occur between childhood and sexual maturation. As a brain matures, half of the synapses are pruned. This process is largely determined by the environment and highly related to the study behavior of the brain [4-7]. It is shown in many studies that synaptic pruning affects the performance of phase synchronization in many diseases. The brains of people with Alzheimer's show desynchronization electrical activity, while synaptic pruning is also dysfunctional [8]. The brains of people with Autism Spectrum Disorder have auditory neural synchronization abnormalities, while synaptic pruning is lacking in the auditory cortex [9]. These studies show that lacking synaptic pruning may make neural synchronization abnormal and finally cause diseases.

The dynamic behaviors of coupled oscillators, especially synchronization, have attracted great attention due to their application in many subjects. Chimera states are 
phenomena characterized by the coexistence of coherent domains (periodic in-phase oscillations) and incoherent domains (characterized by a chaotic behavior in time and space) in the coupling system of identical oscillators $[10,11]$. This phenomenon occurs in coupled FHN neural oscillators [12,13] and relates to many brain functions and diseases [14], including neural bump states [15], uni-hemispheric sleep [16], Parkinson's disease [17], and Alzheimer's disease [18].

A common feature of chimera states is their dependence on the initial conditions. Therefore, the spatial positions of the coherent and incoherent domains are not fixed. Some of the potential mechanisms that may affect the spatial distribution of chimera states in neural networks are explored in the literature. Omelchenko pointed out that modifying coupling with a tweezers method prevents the chimera collapse and also stabilizes the position [19]. Gambuzza found that it is possible to control the position of chimeras by introducing external forcing to the system [20]. Isele noticed that a group of excitable oscillators can attract incoherent structures and repel coherent structures [21]. Ruzzene found that choosing one oscillator and making it link unidirectionally to the other oscillators is the minimal action to control chimeras [22]. Besides the effects on the spatial position of coherent and incoherent domains, coupling structure also affects the excitation and suppression of chimera states [23].

Despite the research mentioned above, the mechanism of how changes in coupling can effectively control the spatial distribution of coherent and incoherent regions is still not clear. Therefore, these theories still haven't been widely applied in neuroscience. This research intends to provide a systematic new comprehension of the relation between synaptic pruning and neural synchronization in the context of chimera states. To the best of our knowledge, this comprehension has never been addressed in previous research. We modified the model of coupled FHN system by changing some of the couplings into unidirectional to illustrate the process of synaptic pruning and focus on the effects of pruning on the spatial distribution of chimera states. The pruning configuration is inspired by the fact that a "pacemaker" with unidirectional couplings can control the position of coherent domains in a small Kuramoto-Sakaguchi network [22]. It is expected that unidirectional couplings combined with a pruned region can control the position better, and such a method can be used to control a network of any size.

The rest of the article is organized as follows: the coupled FHN system and its properties are introduced in Section 2. In Section 3, the effects of a local synchronized region created by the pruning process are investigated. Then, the effects of changing the width of the pruned region are investigated in detail. Furthermore, a brief explanation of this phenomenon is given in Section 4. Finally, the conclusion and some outlooks of this research are given in Section 5.

\section{Model Description}

Proposed initially as a simplification of neural cell activity, the FHN oscillator is a paradigmatic model of excitable systems and is wildly used to describe the dynamic behavior of neurons. Here, a neural network described as N non-locally coupled FHN oscillators is analyzed [12]:

$$
\begin{gathered}
\varepsilon \frac{d u_{k}}{d t}=u_{k}-\frac{u_{k}^{3}}{3}-v_{k}+\frac{\sigma}{2 R} \sum_{j=k-R}^{j=k+R}\left[b_{u u}\left(u_{j}-u_{k}\right)+b_{u v}\left(v_{j}-v_{k}\right)\right] \\
\frac{d v_{k}}{d t}=u_{k}+a+\frac{\sigma}{2 R} \sum_{j=k-R}^{j=k+R}\left[b_{v u}\left(u_{j}-u_{k}\right)+b_{v v}\left(v_{j}-v_{k}\right)\right]
\end{gathered}
$$

where $u_{k}$ and $v_{k}$ are the activator and inhibitor variables, respectively, $\varepsilon>0$ is a timescale parameter, $\sigma$ is the coupling strength among neurons, $a$ is the threshold parameter, and $B$ is the rotational coupling matrix: 


$$
B=\left(\begin{array}{ll}
b_{u u} & b_{u v} \\
b_{v u} & b_{v v}
\end{array}\right)=\left(\begin{array}{cc}
\cos \phi & \sin \phi \\
-\sin \phi & \cos \phi
\end{array}\right)
$$

which is determined only by the coupling phase $\phi$.

Here, all the neurons make up a ring. That is to say, all indices are modulo $N$, where $N$ is the total number of neurons. As it can be seen, each element is coupled to $R$ neighbors on either side. $r=R / N$ is called coupling radius. The value of each parameter mentioned above has its own effect on the performance of the system. $\varepsilon$ and $a$ determine the behavior of a single neuron. $\varepsilon$ determines the slow and fast dynamics associated with $u_{k}$ and $v_{k}$, respectively. $a$ determines whether a neuron exhibits oscillatory $(|a|<1)$ or excitable $(|a|>1)$ behavior [10,24]. $\phi, r$, and $\sigma$ determine the collective behavior of the network. In general, chimera states are expected for a pronounced off-diagonal coupling $(\phi \approx \pi / 2)$, but not for a diagonal one ( $\phi \approx 0$ or $\phi \approx \pi$ ). When changing the value of $r$ and $\sigma$, the number of incoherent domains will change accordingly [12]. To compare with literature, $a, \phi, r, \varepsilon, \sigma$ are chosen to be the same as in Ref. [12]. Namely, $a=0.5, \phi=\pi / 2-0.1, r=0.35, \varepsilon=0.05$, $\sigma=0.1$.

The attractive behaviors of this system are chimera states, which are characterized by the coexistence of coherent domains (periodic in-phase oscillations) and incoherent domains (chaotic behavior in time and space). And the coherent domains can represent phase synchronization in a neural network. However, the spatial distribution of coherent domains is sensitive to the initial condition. This means that there are some mechanisms in neural networks that can control the position of the coherent structures, and synaptic pruning may be one of them.

In order to conduct synaptic pruning that occurs in the neural network, the coupling among neurons is modified. We choose a few contiguous neurons and change their coupling so that the neurons in this region are only affected by each other, but the other neurons in the system are still affected by them. Additionally, the synaptic connections among these neurons are enhanced to keep them synchronizing with each other. In the case of 300 oscillators, a pruned region of 5 oscillators is used. In order to make these 5 oscillators synchronize, the coupling strength between every two nodes in the region is set to 0.2 , while the strength between nodes outside this region is 0.000476 . The single direction coupling strength between nodes inside and outside this region is also 0.000476. In fact, after the coupling strength of the pruned area exceeds a critical value, these oscillators will synchronize, and the increase of the strength will make no difference anymore. The difference in coupling strength between neurons is realistic, considering the diversification of synaptic strength [25]. Though the coupling strength between two neurons outside the pruned region seems very low, the sum of all the couplings of a neuron is still high enough, as such neuron is coupling with more than half of the neurons in the system. The coupling matrix of the neurons is shown in Figure 1. The yellow region is the pruned region, the deep blue region is the non-coupled region, and the light blue region is the region with default coupling strength.

The governing equation of neurons in the pruned region become:

$$
\begin{gathered}
\varepsilon \frac{d u_{k}}{d t}=u_{k}-\frac{u_{k}^{3}}{3}-v_{k}+\sigma_{P} \sum_{j \in P}\left[b_{u u}\left(u_{j}-u_{k}\right)+b_{u v}\left(v_{j}-v_{k}\right)\right] \\
\frac{d v_{k}}{d t}=u_{k}+a+\sigma_{P} \sum_{j \in P}\left[b_{v u}\left(u_{j}-u_{k}\right)+b_{v v}\left(v_{j}-v_{k}\right)\right]
\end{gathered}
$$

where $P$ is the pruned region, $\sigma_{P}$ is the coupling strength among neurons in $P$. The more neurons pruned, the lower the coupling strength required to keep them synchronized.

To solve the differential equations, the 4th order Runge-Kutta method is adopted in this research, with a fixed non-dimensional time step of 0.01 . The initial conditions of the neurons are randomly generated as described in Ref. [12]. 


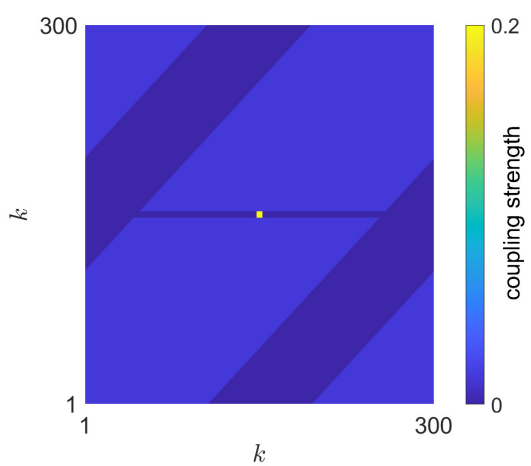

Figure 1. Matrix of coupling strength. Dark blue: not coupled; light blue: default coupling strength; yellow: highly coupled.

\section{Effect of Pruning on Neural Network}

The effect of synaptic pruning on a neural network with 300 neurons is shown in Figure 2 (non-dimensional time $t=1000$ ). The range $148-152$ is the pruned region. It can be seen in Figure 2a that the pruned neurons are synchronized, and they attract the incoherent domain and repel the coherent domain. In Figure $2 b$, it is shown that the phase velocities of neurons in the coherent domain are lower than those in the incoherent domain, and the phase velocities of pruned neurons are much lower than all the others. The neurons that are closest to the pruned region have the highest phase velocities. Figure $2 \mathrm{c}$ shows the local order parameter of the nodes. The parameter is defined as:

$$
Z_{k}=\left|\frac{1}{2 \delta} \sum_{|j-k| \leq \delta} e^{i \Theta_{j}}\right|, k=1, \ldots, N
$$

where $\Theta_{j}=\arctan \left(v_{j} / u_{j}\right)$ denotes the geometric phase of the $\mathrm{j}$-th node. A window size $\delta=5$ is used. A large value of $Z_{k}$ shows that the neuron $k$ is synchronized with its near neighbors, indicating the neuron is in the coherent domain. A small value of $Z_{k}$ means that the neuron $k$ is not synchronized with its near neighbors, indicating the neuron is in the incoherent domain. As the local order parameter's window size is larger than the pruned region, the synchronization of the pruned region can not be observed in Figure 2c, though it can be observed in Figure 2a. For a system without pruning, the coherent domain will be generated at a random place in the system $[19,26]$. In Figure 2c, similarly, the initial position of the coherent domain is not determined. However, the coherent domain is repelled to the opposite position of the pruned region in 500 non-dimensional time. Though this domain drifts back and forth in a very limited region, it is kept away from the pruned region. This phenomenon is counter-intuitive, because one may expect the short synchronized region will be part of the coherent structure, but it turns out to repel the coherent structure. The explanation for such a counter-intuitive phenomenon will be given in Section 4.

The possibility of controlling the positions of chimera states by modifying only one oscillator has been revealed by Ruzzene [22]. Therefore, it is obliged to study if pruning one neuron in an FHN system is sufficient to determine its performance or how the number of pruned neurons affects the system's performance. To make this comparison, four scenarios with different numbers of pruned neurons are simulated $\left(N_{p}=1,2,3,5\right)$, each for 50 cases. Figure 3 shows the average local order parameter in different scenarios.

When only 1 neuron is pruned, as shown in Figure 3a,b, the coherent domain is repelled by the pruned neurons. However, the maximum value of the average local order parameter is much lower than 1 , and it is hard to tell the boundary between the coherent domain and the incoherent domain. This indicates that the coherent structure's position is not the same for all the cases, and the effect on domains' positions is not precise. Figure 4 shows an example that the pruned neuron failed to repel the coherent domain in 1000 nondimensional time. When 2 or 3 neurons are pruned, as shown in Figure $3 b, c$, the values of $Z_{k}$ on two sides of the figure get higher. The boundaries between different regions are 
clearer. This indicates that the coherent structure's positions are similar for most of all the 50 cases. When 5 neurons are pruned, as shown in Figure 3d, the values of $Z_{k}$ on two sides of the figure are very close to 1 , and the boundaries between different regions are very clear. This indicates that the coherent structure's positions are the same for all 50 cases.

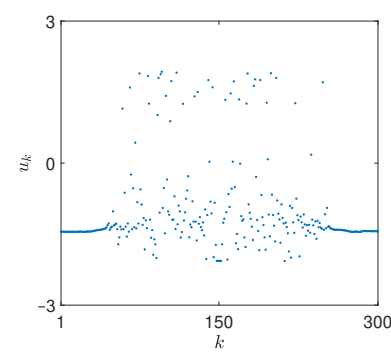

(a)

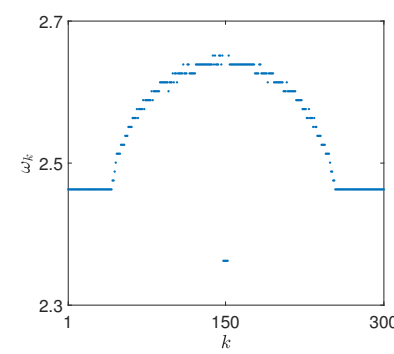

(b)

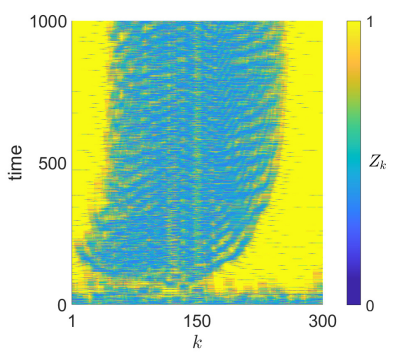

(c)

Figure 2. Effect of synaptic pruning on a FHN system. Neurons 148-152 are pruned. The coherent domain is repelled by the pruned region. (a) Snapshot of the variables $u_{k}$ for $t=1000$, (b) mean phase velocities $\omega_{k},(\mathbf{c})$ local order parameter $Z_{K}$. Parameters: $N=300, r=0.35, \sigma=0.1, \sigma_{P}=0.2$, $a=0.5, \phi=\pi / 2-0.1$.

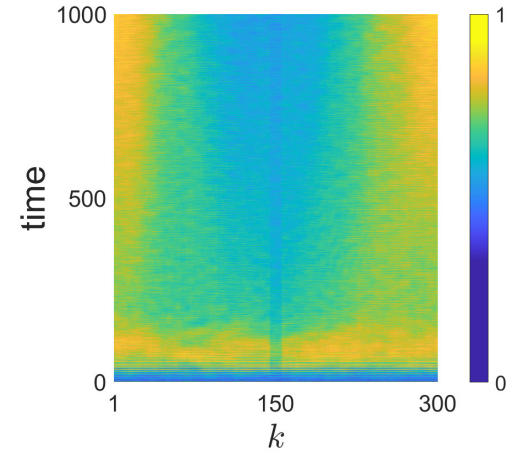

(a)

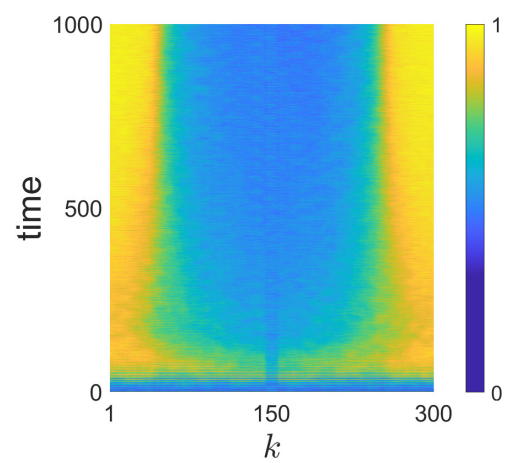

(c)

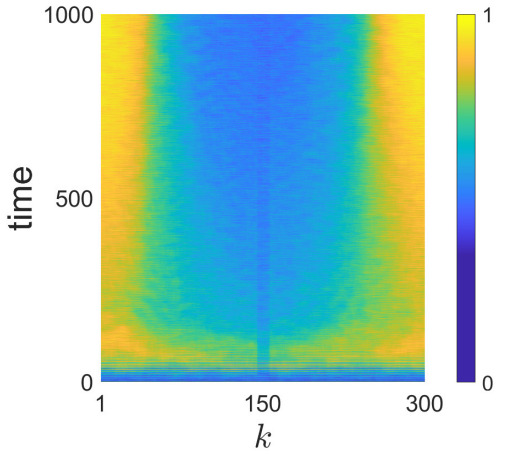

(b)

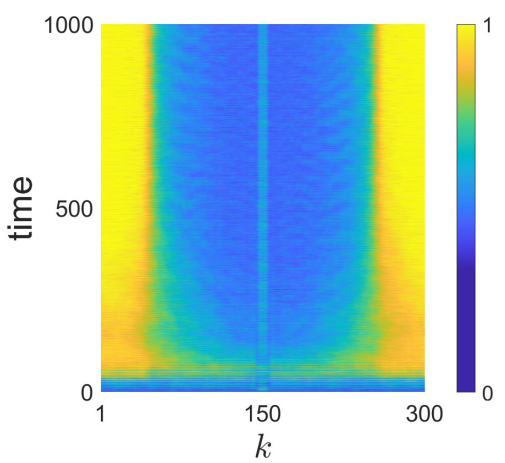

(d)

Figure 3. The number of pruned neurons affects the performance of the system. (a) Average local order parameter of 50 cases when $N_{p}=1$ (node 150), (b) $N_{p}=2$ (nodes 150 to 151), (c) $N_{p}=3$ (nodes 149 to 151 ), (d) $N_{p}=5$ (nodes 148 to 152). $N=300$, other parameters are the same as in Figure 2.

To illustrate the impact of the number of pruned neurons with more detail, the probability distribution of the position of the coherent domain is plotted in Figure 5. The coherent region's beginning and ending nodes are extracted for each simulation. Then, the number of times for a node located in the coherent region can be counted. Finally, the corresponding probability is obtained. When only one neuron is pruned, the coherent structure may locate at any position, even next to the pruned region. The probability for the first neuron on the ring to locate in the coherent structure is only $50 \%$. As more neurons are pruned, 
the distribution of coherent structure is more uniform. When 5 neurons are pruned, the coherent structure can only be located opposite the pruned region. Namely, the position is precisely controlled.

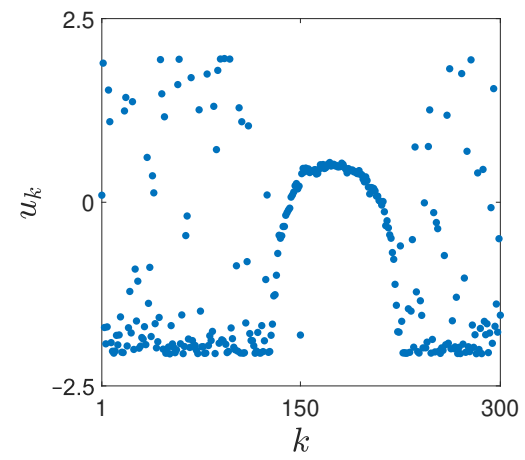

(a)

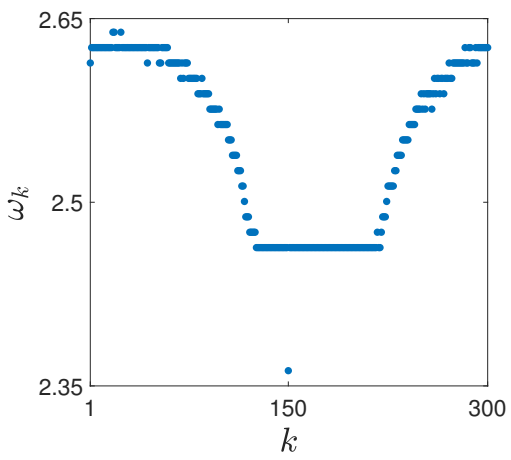

(b)

Figure 4. Pruning one neuron is not effective enough to control the position of chimera states. (a) Snapshot of $u_{k}$ for $t=1000$, (b) mean phase velocities $\omega_{k}$.

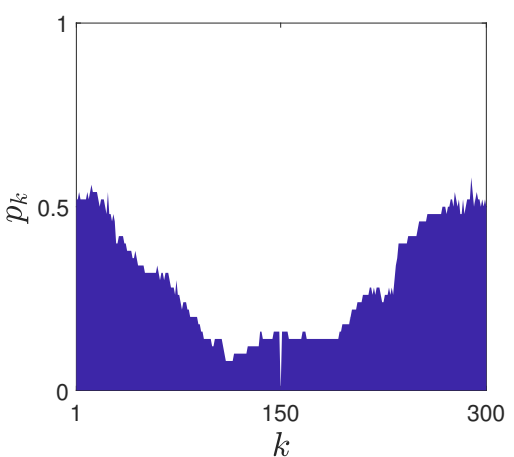

(a)

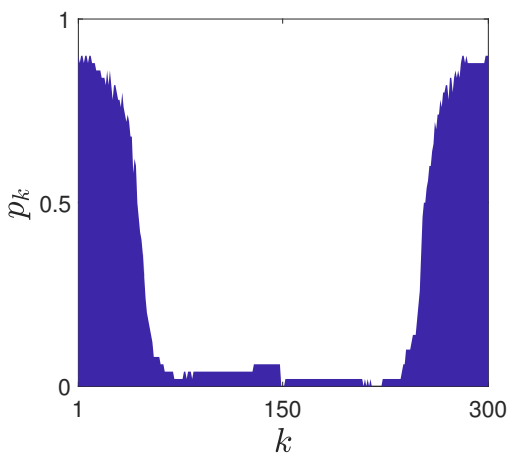

(c)

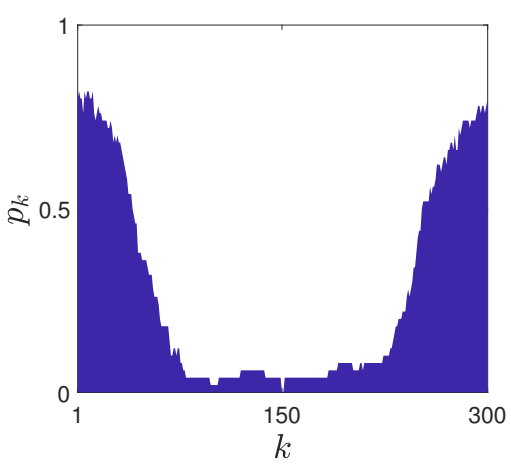

(b)

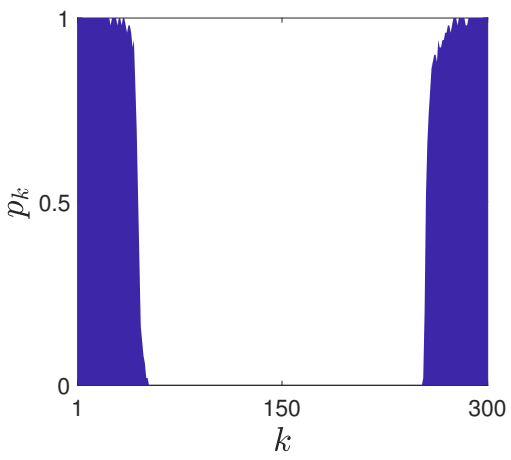

(d)

Figure 5. The number of pruned neurons affects the performance of the system. (a) Probability distribution of the position of coherent domain when $N_{p}=1$ (node 150), (b) $N_{p}=2$ (nodes 150 to 151), (c) $N_{p}=3$ (nodes 149 to 151), (d) $N_{p}=5$ (nodes 148 to 152). $N=300$, other parameters are the same as in Figure 2.

Though it may still be possible that pruning one neuron is sufficient to determine the position of the coherent domain after a process of an extremely long time, it can not be relevant to any phase synchronization that occurs in brain activities. Because 1000 non-dimensional time is already equivalent to several seconds or minutes, more than the time of enhancement of phase synchronization detected in brain activities. 


\section{Mechanism Explanation}

To explain the phenomena observed, the simplest coupled-FHN system, namely the system with only two oscillators, is considered. Figure 6 shows the dynamic behavior of an FHN oscillator coupled with another free-oscillating FHN oscillator. $\varepsilon=0.05$, $a=0.5, \phi=\pi / 2-0.1$, the strength of the unidirectional coupling $\sigma=0.1$. The phases of the oscillators are described as $\theta=\arctan (v, u)$. The initial values of $\theta$ are both in the interval $(-\pi, \pi]$, and they increase monotonically. Two points on the trajectory of a free FHN oscillator with a time difference of 0.30 are used as the initial conditions of the two oscillators. The initial phase difference of the oscillators is $\Delta \theta=2.055$ in Figure 6a, and $\Delta \theta=2.182$ in Figure $6 \mathrm{~b}$.

As shown in Figure 6a, when a coupled oscillator is leading the free oscillator, it will be accelerated. The phase difference between them will keep increasing and finally be $2 \pi$, which means the coupled oscillator catches up with the free one again.

However, the case is different if the coupled oscillator is falling behind, as shown in Figure $6 \mathrm{~b}$. The coupled oscillator will still be accelerated, but the phase difference will keep decreasing and finally be 0 . The different effects on positive and negative phase difference is a kind of symmetry breaking, which is one of the reasons that caused chimera states.

From the cases of two coupled oscillators above, it can be known that when one FHN oscillator is coupled with another, there is a phase difference between them, and if the coupling strength is chosen properly, the coupling may always accelerate this oscillator and increase its phase velocity. This explains why the frequencies of all the neurons in the network are higher than their natural frequencies. This is a combined effect of the nonlinearity of a single oscillator and the coupling between oscillators, and this may explain why chimera states only occur when coupling strength is in a specific range.

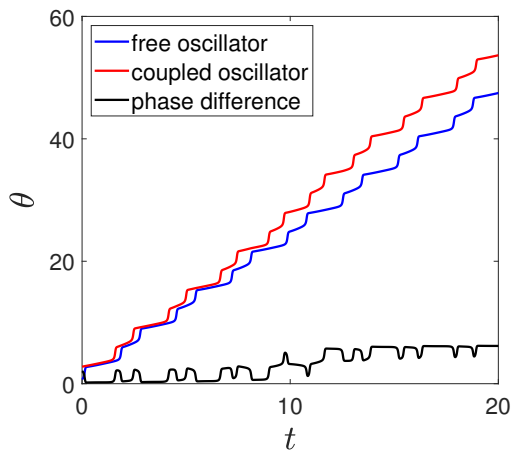

(a)

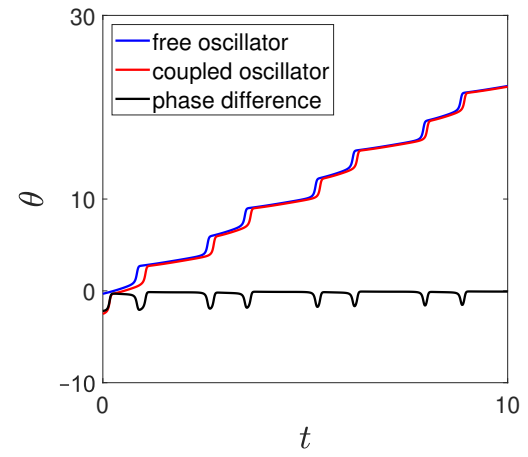

(b)

Figure 6. The effect of unidirectional coupling between two FHN oscillators. (a) The coupled oscillator leads the free oscillator, $(\mathbf{b})$ the coupled oscillator falls behind the free oscillator.

In the ring-shaped neural network, the initial phases of all the neurons are random. Some neurons' phases can be leading the average of their neighbors, so their phase velocities will be accelerated by their neighbors, and when their phases are almost $2 \pi$ ahead of their neighbors, they can be regarded as falling behind, while some other neurons become leading and accelerated. The neurons in the pruned region synchronize in a short time, and their phase velocities become fixed. However, for the other neurons, there will always be some leading ones, so the process of mutual acceleration will never end, and the neurons cannot all synchronize. In this process, synchronized neurons gradually form a coherent domain. As these neurons hardly have phase differences with their neighbors, they will hardly be accelerated and have lower phase velocities. On the other hand, desynchronized neurons always have phase differences with their neighbors, therefore largely accelerated and have higher phase velocities. These neurons finally form an incoherent domain. As the phase velocities of neurons in the pruned region are much lower than all the other neurons, there will always be phase differences between these neurons and the neurons located near this region. Therefore, the neurons near the pruned region will be accelerated the most and 
finally get the highest phase velocities. That is to say, the center of the incoherent structure will drift to this region.

\section{Conclusions and Outlook}

This research investigates the effect of pruning on a ring-shape-FHN-system with chimera states, which explains how synaptic pruning affects the spatial distribution of synchronized and desynchronized regions in neural networks on a theoretically and numerically sound basis. The approach of the present research is not seen in the current literature. The pruning process is conducted by modifying the coupling in a small region of the network and making the pruned neurons synchronize. The impact of the number of pruned neurons on the performance of the system is also investigated. For a system with 300 neurons, pruning 5 neurons is sufficient to control the spatial position of the coherent domain precisely and fast. Moreover, the mechanism of this method is illustrated. The pruned neurons accelerate their neighbors so that the center of the incoherent structure is attracted.

Considering that FHN systems widely exist in many neuroscience and engineering fields, the conclusion of this research can find many potential applications. One example is that in the multi-link network with bio-inspired motor neuron dynamical unit [24,27], pruning can be used to easily generate rhythmic (coherent) and chaotic (incoherent) dynamics. Rhythmic patterns can be adopted in locomotion control, and chaotic dynamics are useful when searching reflexes are triggered only in a subpart of the network.

As shown in the explanation of the phenomenon, the neurons will be accelerated by coupling with the pruned neuron when there is a phase difference. Therefore, it is natural to think about what would happen if the phase velocity of the neurons is also modified in the pruned region, especially if it is the same as the phase velocity in the coherent domain.

The mechanism of pruning found in the research shows a type of symmetry breaking in a coupled identical oscillator system, which illustrates the possibility of complicated collective behavior based on simple individual behavior. This differentiation caused by symmetry breaking may explain how neurons in a network are divided into groups with different functions.

Author Contributions: Z.Z. conducted the conceptual study, formal analysis and investigation. Both the authors contributed to method development, paper review and writing. L.D. supervised the research. All authors have read and agreed to the published version of the manuscript.

Funding: This research was funded bythe Natural Sciences and Engineering Research Council of Canada (NSERC), Mathematics of Information Technology and Complex Systems (Mitacs), and Wuhan Polytechnic University grant number WGZ202000011.

Data Availability Statement: The data presented in this study are available on request from the corresponding author.

Conflicts of Interest: The authors declare no conflict of interest.

\section{References}

1. Fell, J.; Axmacher, N. The role of phase synchronization in memory processes. Nat. Rev. Neurosci. 2011, 12, 105-118. [CrossRef] [PubMed]

2. Kveraga, K.; Ghuman, A.S.; Kassam, K.S.; Aminoff, E.A.; Hämäläinen, M.S.; Chaumon, M.; Bar, M. Early onset of neural synchronization in the contextual associations network. Proc. Natl. Acad. Sci. USA 2011, 108, 3389-3394. [CrossRef] [PubMed]

3. Guevara Erra, R.; Perez Velazquez, J.L.; Rosenblum, M. Neural Synchronization from the Perspective of Non-linear Dynamics. Front. Comput. Neurosci. 2017, 11, 98. [CrossRef] [PubMed]

4. Lieberman, O.J.; McGuirt, A.F.; Tang, G.; Sulzer, D. Roles for neuronal and glial autophagy in synaptic pruning during development. Neurobiol. Dis. 2019, 122, 49-63. [CrossRef]

5. Sakai, J. Core Concept: How synaptic pruning shapes neural wiring during development and, possibly, in disease. Proc. Natl. Acad. Sci. USA 2020, 117, 16096-16099. [CrossRef]

6. Millán, A.P.; Torres, J.J.; Johnson, S.; Marro, J. Concurrence of form and function in developing networks and its role in synaptic pruning. Nat. Commun. 2018, 9, 2236. [CrossRef] 
7. Paolicelli, R.C.; Bolasco, G.; Pagani, F.; Maggi, L.; Scianni, M.; Panzanelli, P.; Giustetto, M.; Ferreira, T.A.; Guiducci, E.; Dumas, L.; et al. Synaptic Pruning by Microglia Is Necessary for Normal Brain Development. Science 2011, 333, 1456-1458. [CrossRef]

8. Aron, L.; Yankner, B.A. Neural synchronization in Alzheimer's disease. Nature 2016, 540, 207-208. [CrossRef]

9. De Stefano, L.A.; Schmitt, L.M.; White, S.P.; Mosconi, M.W.; Sweeney, J.A.; Ethridge, L.E. Developmental Effects on Auditory Neural Oscillatory Synchronization Abnormalities in Autism Spectrum Disorder. Front. Integr. Neurosci. 2019, 13, 34. [CrossRef]

10. Schöll, E. Synchronization patterns and chimera states in complex networks: Interplay of topology and dynamics. Eur. Phys. J. Spec. Top. 2016, 225, 891-919. [CrossRef]

11. Panaggio, M.J.; Abrams, D.M. Chimera states: Coexistence of coherence and incoherence in networks of coupled oscillators. Nonlinearity 2015, 28, R67-R87. [CrossRef]

12. Omelchenko, I.; Omel'chenko, O.E.; Hövel, P.; Schöll, E. When Nonlocal Coupling between Oscillators Becomes Stronger: Patched Synchrony or Multichimera States. Phys. Rev. Lett. 2013, 110, 224101. [CrossRef] [PubMed]

13. Omelchenko, I.; Provata, A.; Hizanidis, J.; Schöll, E.; Hövel, P. Robustness of chimera states for coupled FitzHugh-Nagumo oscillators. Phys. Rev. E 2015, 91, 022917. [CrossRef]

14. Majhi, S.; Bera, B.K.; Ghosh, D.; Perc, M. Chimera states in neuronal networks: A review. Phys. Life Rev. 2019, $28,100-121$. [CrossRef] [PubMed]

15. Laing, C.R. Fronts and bumps in spatially extended Kuramoto networks. Phys. D Nonlinear Phenom. 2011, $240,1960-1971$. [CrossRef]

16. Haugland, S.W.; Schmidt, L.; Krischer, K. Self-organized alternating chimera states in oscillatory media. Sci. Rep. 2015, 5, 9883. [CrossRef]

17. von Papen, M.; Dafsari, H.; Florin, E.; Gerick, F.; Timmermann, L.; Saur, J. Phase-coherence classification: A new wavelet-based method to separate local field potentials into local (in)coherent and volume-conducted components. J. Neurosci. Methods 2017, 291, 198-212. [CrossRef]

18. Caravaglios, G.; Muscoso, E.G.; Di Maria, G.; Costanzo, E. Patients with mild cognitive impairment have an abnormal upperalpha event-related desynchronization/synchronization (ERD/ERS) during a task of temporal attention. J. Neural Transm. 2015, 122, 441-453. [CrossRef]

19. Omelchenko, I.; Omel'chenko, O.E.; Zakharova, A.; Wolfrum, M.; Schöll, E. Tweezers for Chimeras in Small Networks. Phys. Rev. Lett. 2016, 116, 114101. [CrossRef]

20. Gambuzza, L.V.; Frasca, M. Pinning control of chimera states. Phys. Rev. E 2016, 94, 022306. [CrossRef]

21. Isele, T.; Hizanidis, J.; Provata, A.; Hövel, P. Controlling chimera states: The influence of excitable units. Phys. Rev. E 2016, 93, 022217. [CrossRef] [PubMed]

22. Ruzzene, G.; Omelchenko, I.; Schöll, E.; Zakharova, A.; Andrzejak, R.G. Controlling chimera states via minimal coupling modification. Chaos Interdiscip. J. Nonlinear Sci. 2019, 29, 051103. [CrossRef]

23. Maksimenko, V.A.; Makarov, V.V.; Bera, B.K.; Ghosh, D.; Dana, S.K.; Goremyko, M.V.; Frolov, N.S.; Koronovskii, A.A.; Hramov, A.E. Excitation and suppression of chimera states by multiplexing. Phys. Rev. E 2016, 94, 052205. [CrossRef] [PubMed]

24. Arena, P.; Patanè, L.; Spinosa, A.G. A New Embodied Motor-Neuron Architecture. IEEE Trans. Control. Syst. Technol. 2021, 1-8. [CrossRef]

25. Atwood, H.L.; Karunanithi, S. Diversification of synaptic strength: Presynaptic elements. Nat. Rev. Neurosci. 2002, 3, 497-516. [CrossRef] [PubMed]

26. Abrams, D.M.; Strogatz, S.H. Chimera States for Coupled Oscillators. Phys. Rev. Lett. 2004, 93, 174102. [CrossRef] [PubMed]

27. Arena, P.; Patané, L.; Spinosa, A.G. A nullcline-based control strategy for PWL-shaped oscillators. Nonlinear Dyn. 2019, 97, 1011-1033. [CrossRef] 